\title{
Erratum: Modelling the Effects of Operating Conditions on Motor Power Consumption in Single Screw Extrusion
}

\author{
Chamil Abeykoon ${ }^{1, *}$, Marion McAfee ${ }^{2}$, Kang Li ${ }^{3}$, Peter J. Martin ${ }^{1}$, \\ Jing Deng ${ }^{3}$, and Adrian L. Kelly ${ }^{4}$ \\ ${ }^{1}$ School of Mechanical and Aerospace Engineering, \\ Queen's University Belfast, Belfast, BT9 5AH, UK \\ Tel.: $+44289097236,+442890974164$ \\ yabeykoon01@qub.ac.uk \\ 2 Department of Mechanical and Electronic Engineering, \\ Institute of Technology Sligo, Sligo, Ireland \\ ${ }^{3}$ School of Electronics, Electrical Engineering and Computer Science, \\ Queen's University Belfast, Belfast, BT9 5AH, UK \\ ${ }^{4}$ IRC in Polymer Science and Technology, School of Engineering, \\ Design and Technology, University of Bradford, Bradford, BD7 1DP, UK
}

DOI 10.1007/978-3-642-15597-0_57

Some of the reference tags in this paper are displayed incorrectly:

"[3]" on page 10 should be "Kent [3]"

"[4]" on page 10 should be "Falkner [4]"

"[7]" on page 10 should be "Rasid and Wood [7]"

"[9]" on page 11 should be "Lai and Yu [9]"

"[10]" on page 11 should be "Wilczynski [10]" 
The equations 3 and 4 along with their description on page 15 are also displayed incorrectly:

$$
M E=y(t)-\hat{y}(t)
$$

$N P P E \triangleq\left[\sum_{t=1}^{N}(\hat{y}(t)-y(t))^{2} / \sum_{t=1}^{N} y(t)^{2}\right]^{1 / 2} \times 100 \%$

Where $y(t)$ is the measured motor power consumption and the $\hat{y}(t)$ is the model estimated motor power consumption.

should be :

$$
M E=y_{i}-\hat{y}_{i}
$$

$N P P E \triangleq\left[\sum_{i=1}^{n}\left(\hat{y}_{i}-y_{i}\right)^{2} / \sum_{i=1}^{n} y_{i}^{2}\right]^{1 / 2} \times 100 \%$

Where $y_{i}$ is the measured motor power consumption, the $\hat{y}_{i}$ is the model estimated motor power consumption, and $n$ is the number of data points. 